\title{
Improving 4DSTEM measurements of atomic charge and electrostatic potential via energy filtration
}

\author{
Thomas Pekin ${ }^{1}$, Marcel Schloz ${ }^{1}$, Benedikt Haas², Wouter Van den Broek ${ }^{3}$ and Christoph Koch ${ }^{2}$ \\ ${ }^{1}$ Humboldt Universität zu Berlin, United States, ${ }^{2}$ Department of Physics, Humboldt University of Berlin, \\ Berlin, Germany, United States, ${ }^{3}$ Humboldt Universität zu Berlin, Berlin, Berlin, United States
}

In the past few years, data acquired using four dimensional scanning electron microscopy (4DSTEM) has been used to measure and map atomic potentials with unprecedented resolution and sensitivity to light elements in 2D, or quasi-2D materials [1-3]. These results either rely on integrated center of mass measurements (iCOM) [4] or on ptychographic reconstruction algorithms. iCOM is a conceptually simple technique in which the center of mass of the forward scattered beam is measured as the beam is rastered across the sample, and then converted into electric field, charge density or atomic potential, while the more complex and computationally intensive ptychographic algorithms optimize the atomic potential, among other parameters, to best fit a forward scattering model to the acquired data [5-7]. These other parameters can include finite thickness with the introduction of multislice algorithms, partial coherence, uncertainty with respect to electron probe position on the sample and aberrations in the probe itself, at the cost of increased computation times and more hyperparameters to modify. While ptychography can model many of these non-ideal experimental settings, the reconstruction algorithms cannot yet appropriately model inelastic electron scattering events, which is an increasing percentage of all electrons as the sample thickness increases and therefore a limit on the reconstruction quality. In order to effectively reconstruct atomic potentials in thicker samples then, these electrons must either be accounted for in the forward scattering model, or removed altogether during the experiment.

Here we will present an approach to remove the majority of inelastically scattered electrons during 4DSTEM experiments at atomic resolution and compare results obtained by iCOM and ptychography on both thick and thin samples. By using the energy filter in the spectrometer on a Nion HERMES microscope, a cutoff at $\sim 5 \mathrm{eV}$ energy loss in 4DSTEM datasets can be implemented. This energy cutoff removes the majority of the bulk plasmon losses for many materials. The experiments shown below were performed at an accelerating voltage of $60 \mathrm{kV}$, and the data was acquired with a direct electron detector that can be operated up to 18,000 frames per second, both of which can reduce damage to the sample.

Figure 1 shows a comparison between iCOM results of twisted bilayer graphene (TBG) from a 4DSTEM dataset with and without energy filtering. In both examples can the atomic Moire pattern be clearly resolved, and there is no appreciable improvement in the iCOM results when the electrons are filtered. In fact, that is to be expected, as only about $4 \%$ of the electrons that are scattered by the sample are removed, which can be calculated directly from the images obtained, and used as a metric for when energy filtering might be beneficial.

Figure 2 shows the same comparison but the atomic potentials have been recovered via ptychographic reconstruction, where we have optimized the object and probe. For the sake of reconstruction time, only the central $3 \mathrm{~nm}$ from Fig. 1 have been reconstructed, and we have not optimized probe positions or partial coherence, but these would further enhance the reconstruction fidelity. Again, it is difficult to compare reconstruction quality between unfiltered and filtered reconstructions directly, but in panels $\mathrm{c}$ and $\mathrm{d}$ where the FFT of the data has been calculated, the spots corresponding to the misorientation angle between the bilayers are better resolved in the filtered data when compared to equivalent spots in the unfiltered data. As there are a variety of explanations for why this may be the case (region of sample from which the FFT was computed, for example), a more complete comparison will be presented in person. 
Following these initial results, we will show comparisons between results obtained with thick and thin samples as well, with up to $50 \%$ of the electrons removed. We expect that the results will show that iCOM is robust to the influence of inelastically scattered electrons in both thick and thin samples, and while ptychography of thin samples will be similarly robust when the amount of filtered electrons is small, that energy filtration will be key to reconstructing the atomic potentials of thicker samples when larger percentages of electrons are removed.
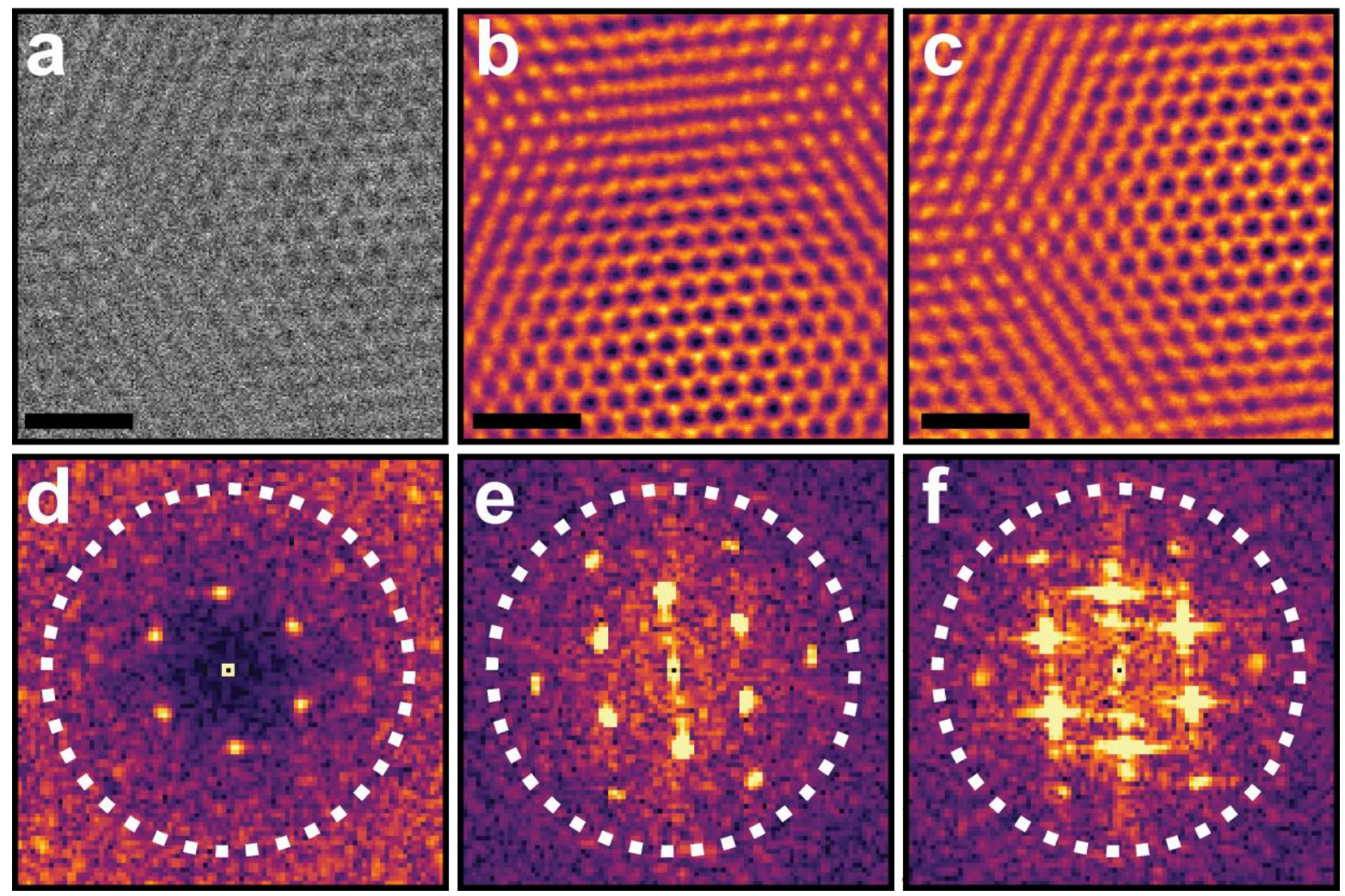

Figure 1. a) Simultaneously acquired HAADF corresponding to c, of twisted bilayer graphene. The scan width is $4 \mathrm{~nm}$ and the scale bar corresponds to $1 \mathrm{~nm}$. b) Atomic potentials as measured with iCOM, without energy filtering, and c) with energy filtering. Note that these are roughly the same area, but with some sample drift towards the top right. d-f) FFTs of the images above, with a dotted circle marking $1 \AA$ resolution. The results between $\mathrm{b}$ and $\mathrm{c}$, and $\mathrm{e}$ and $\mathrm{f}$ are roughly identical, with streaking in $\mathrm{f}$ from the centering (shifting) operation of the high resolution section of the image to the center. 


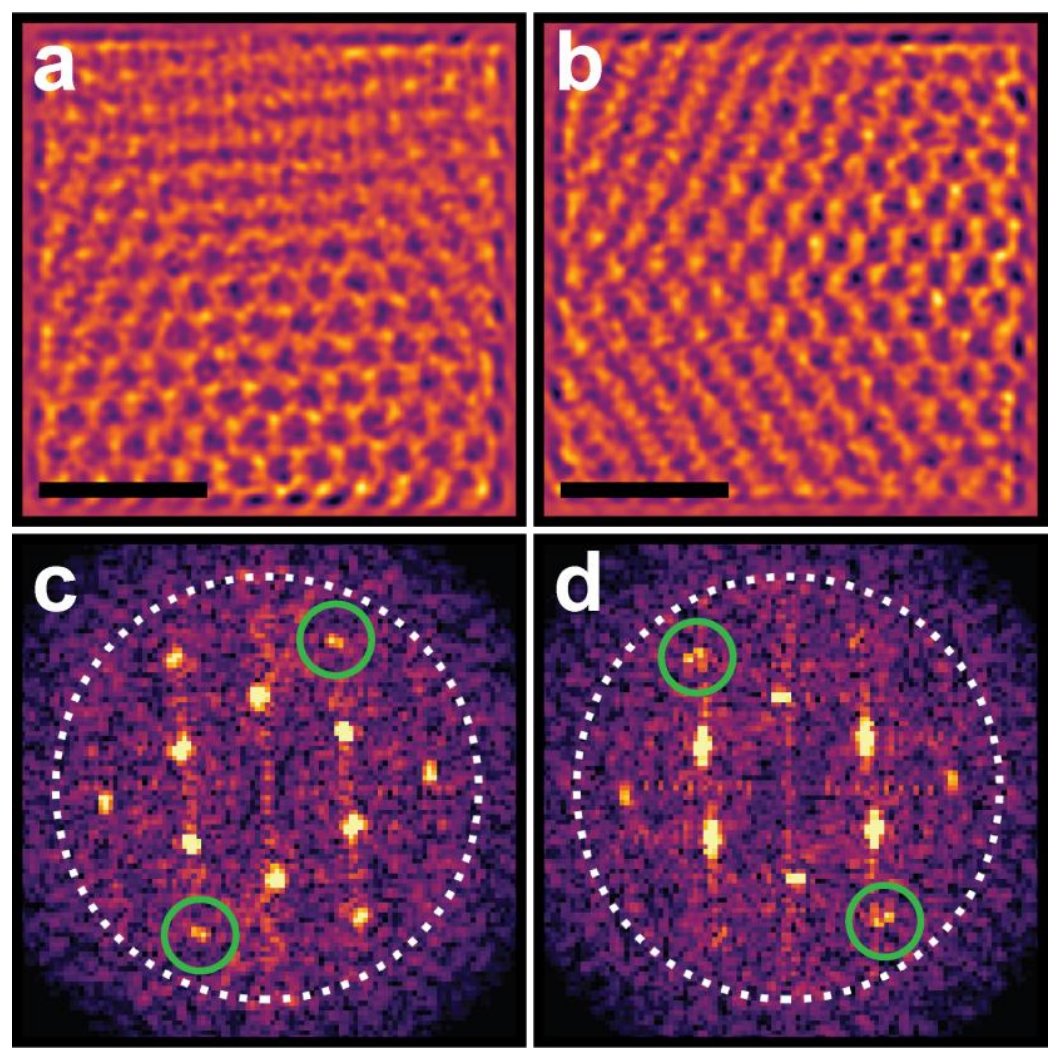

Figure 2. 2a) Ptychographic reconstructions of the central $3 \mathrm{~nm}$ of Fig. 1b, which used unfiltered data. b) Ptychographic reconstruction of the central $3 \mathrm{~nm}$ of Fig. 1c, which used energy filtered data. Both scale bars are $1 \mathrm{~nm}$. c and d) The Fourier transforms of a) and b), respectively, with the dotted circle marking $1 \AA$ resolution. Note that the reconstruction quality remains about the same, but the dumbbells (circled in green) corresponding to the misorientation of the bilayer in d) are slightly better resolved than in c).

\section{References}

1. Rodenburg, John M. "Ptychography and related diffractive imaging methods." Advances in imaging and electron physics 150 (2008): 87-184.

2. Jiang, Yi, et al. "Electron ptychography of 2D materials to deep sub-ångström resolution." Nature 559.7714 (2018): 343-349.

3. Yang, H., et al. "Simultaneous atomic-resolution electron ptychography and Z-contrast imaging of light and heavy elements in complex nanostructures." Nature Communications 7.1 (2016): 1-8.

4. Hachtel, Jordan A., Juan Carlos Idrobo, and Miaofang Chi. "Sub-Ångstrom electric field measurements on a universal detector in a scanning transmission electron microscope." Advanced Structural and Chemical Imaging 4.1 (2018): 1-10.

5. Schloz, Marcel, et al. "Overcoming information reduced data and experimentally uncertain parameters in ptychography with regularized optimization." Optics Express 28.19 (2020): 28306-28323.

6. Chen, Zhen, et al. "Mixed-state electron ptychography enables sub-angstrom resolution imaging with picometer precision at low dose." Nature Communications 11.1 (2020): 1-10.

7. Li, Peng, and Andrew Maiden. "Multi-slice ptychographic tomography." Scientific Reports 8.1 (2018): 110 . 